\title{
Successful percutaneous coronary intervention for multiple coronary artery aneurysms causing acute inferior myocardial infarction
}

\author{
Tsuyoshi Honda, Shunichi Koide, Tomokazu Ikemoto, Ryusuke Tsunoda, Teruhiko Ito, Hiromi Yoshimura \\ Department of Cardiovascular Medicine, Japanese Red Cross Kumamoto Hospital, Kumamoto, Japan
}

Adv Interv Cardiol 2021; 17, 1 (63): 112-113

DOI: https://doi.org/10.5114/aic.2021.104778

A 59-year-old man was referred to our hospital for chest pain he had been experiencing for several hours. The electrocardiogram showed ST-segment elevation in leads II, III, aVF, V4-V6. Emergent coronary angiography (CAG) showed a total occlusion of the proximal right coronary artery (RCA) with multiple calcified coronary artery aneurysms (CAAs) (Figure $1 \mathrm{~A}$ ). There was no significant lesion of the left coronary artery.

The RCA was intubated with a 7-Fr Amplatz-left 1.0 guiding catheter (Launcher; Medtronic, USA). A 0.014 guidewire (Runthrough NS Floppy; Terumo, Japan) could not pass through the lesion. We switched to the 0.014 guidewire (Conquest Pro12; Asahi Intecc, Japan). Supported with a microcatheter (Caravel MC; Asahi Intecc, Japan) the guidewire passed through the lesion in a loop (Figure $1 \mathrm{~B}$ ). Ballooning with a $3.0 \times 30 \mathrm{~mm}$ catheter (Euphora; Medtronic, USA) was performed with post-dilatation using a $4.0 \times 10 \mathrm{~mm}$ catheter (Raiden3; Kaneka, Japan). The balloon did not fully expand in the lesion, so stenting was not attempted. We performed thrombectomy using a 7-Fr catheter (ThrombusterlII-GR, Kaneka, Japan) more than 10 times and a large amount of red thrombus was obtained. Although we confirmed TIMI-3 flow after repeating thrombectomy and ballooning, some residual thrombus was still noted on CAG (Figure $1 \mathrm{C})$. Therefore we decided to administer intracoronary 120,000 units of urokinase. We confirmed resolution of his chest pain and ST-segment elevation, and preserved left ventricle (LV) function. Although we offered low-dose aspirin and warfarin, aspirin was stopped due to minor bleeding 14 months later.

After 18 months coronary computed tomography (CT) was performed to assess patency and morphology of the
CAAs (Figure $1 \mathrm{D}$ ). Although coronary bypass surgery was offered to him, he refused to undergo surgery.

CAAs are defined as dilatation of coronary artery diameter $\geq 1.5$ times its original size and are reported to have an incidence of $0.3-5 \%$ in angiographic series [1, 2]. Atherosclerosis is the most common cause of CAAs and other etiologies of CAAs are: congenital, complicated percutaneous coronary intervention (PCI), Kawasaki's disease, and Marfan syndrome [1]. The cause of CAAs in this case is unknown because the patient does not have any past histories. $\mathrm{PCl}$ of an aneurysmal culprit vessel in the setting of acute myocardial infarction (AMI) is associated with lower procedural success and higher incidence of no-reflow and distal embolization [2]. It is difficult to assess the sizing and landing zone of the stent to be deployed at an aneurysmal vessel in consideration of the risk of stent thrombosis and stent migration [2].

Patients who are asymptomatic or have no significant coronary atheromatous disease can be considered good candidates for conservative therapy, the principal goal of which is to prevent formation of any thrombus inside the aneurysm. This goal can be by attained by treating this population of patients with anticoagulation therapy with follow-up every 3 months [3, 4].

In conclusion, the presented case with multiple CAAs causing $\mathrm{AMI}$ is rare and the treatment depends on the clinical findings, location, and size of the CAAs. Because primary $\mathrm{PCl}$ involving CAAs is challenging, causing difficulties in "wiring" the distal part of the lesion, it might be useful to wire with a loop by using a microcatheter.

\section{Conflict of interest}

The authors declare no conflict of interest.

\section{Corresponding author:}

Tsuyoshi Honda PhD, Department of Cardiovascular Medicine, Japanese Red Cross Kumamoto Hospital, Kumamoto, Japan, phone: +48 81-96-384-2111, e-mail: tsuyohonda-circ@umin.ac.jp Received: 10.12.2020, accepted: 28.01.2021. 

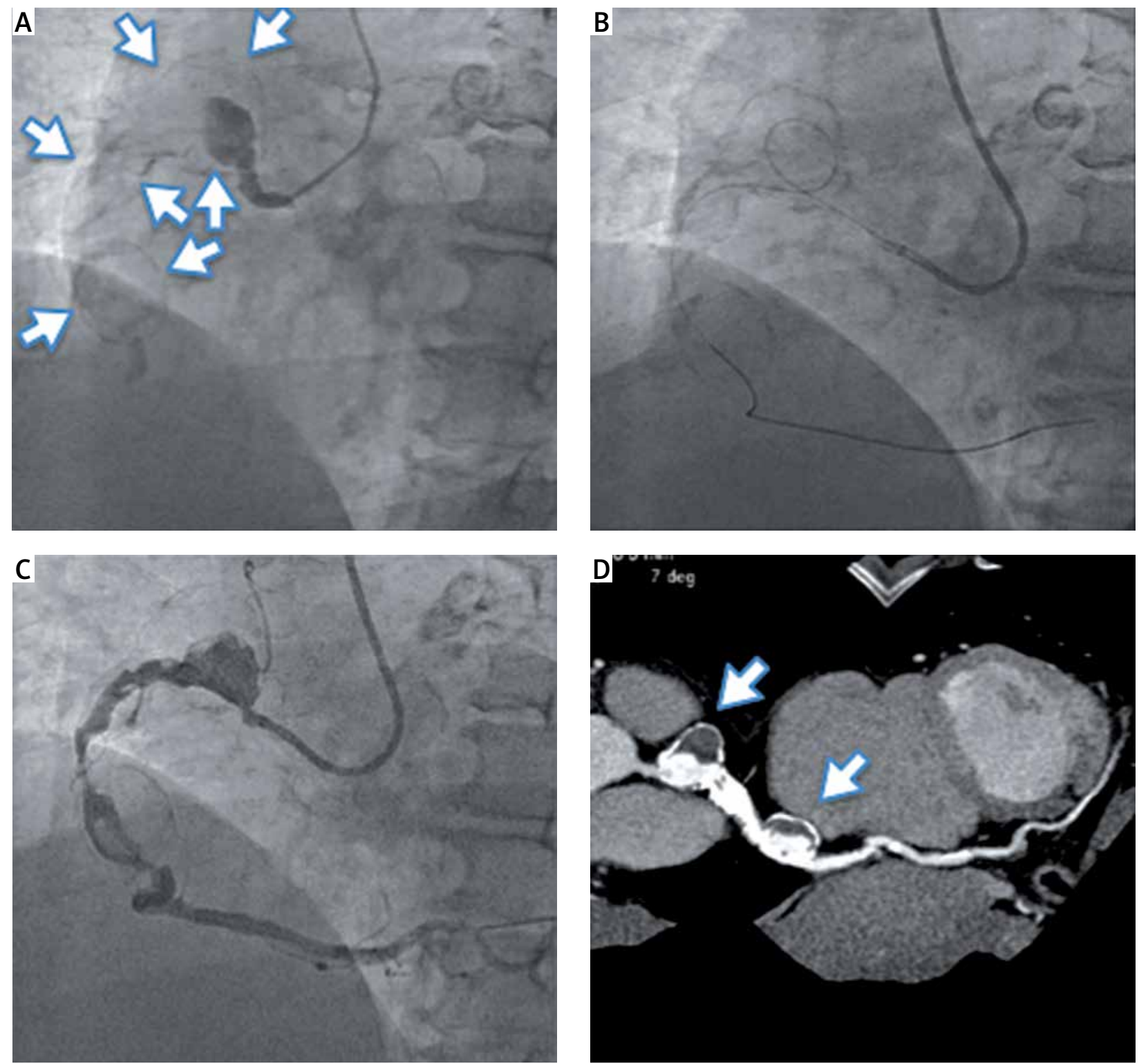

Figure 1. coronary angiography (CAG) before and after percutaneous coronary intervention (PCI), and follow-up computed tomography (CT). A - Right CAG before $\mathrm{PCl}$, white arrows: multiple coronary artery aneurysms, B - right CAG after wire cross, C - right CAG after $\mathrm{PCI}, \mathrm{D}-\mathrm{CT}$ angiography 18 months later, white arrows: multiple coronary artery aneurysms

\section{References}

1. Núñez-Gil IJ, Cerrato E, Bollati M, et al. Coronary artery aneurysms, insights from the international coronary artery aneurysm registry (CAAR). Int J Cardiol 2020; 299: 49-55.

2. Kawasara A, Núñez Gil IJ, Alqahtani F, et al. Management of coronary artery aneurysms. JACC Cardiovasc Interv 2018; 11: 1211-23.

3. Kim JH, Park HB, Lee YB, et al. Large coronary artery aneurysm with thrombotic coronary occlusion resulting in ST-elevation myocardial infarction after warfarin interrruption. J Lipid Atheroscler 2014; 3: 105-9.

4. Khouzam RN, Soufi MK, Whitted A. Saccular aneurysm and stenosis of the left descending artery presenting with acute coronary syndrome. What is the best treatment: CABG or PCI? J Cardiol Cases 2013; 8: 129-30. 